\title{
Laboratory experiments on rain-driven convection: implications for planetary dynamos
}

\author{
Peter Olson*, Maylis Landeau, \& Benjamin H. Hirsh \\ Department of Earth \& Planetary Sciences \\ Johns Hopkins University, Baltimore, MD 21218
}

\begin{abstract}
Compositional convection driven by precipitating solids or immiscible liquids has been invoked as a dynamo mechanism in planets and satellites throughout the solar system, including Mercury, Ganymede, and the Earth. Here we report laboratory experiments on turbulent rain-driven convection, analogs for the flows generated by precipitation within planetary fluid interiors. We subject a two-layer fluid to a uniform intensity rainfall, in which the rain is immiscible in the upper layer and miscible in the lower layer. Rain falls through the upper layer and accumulates as a two-fluid emulsion in the interfacial region between the layers. In experiments where the rain is denser than the lower fluid, rain-injected vortices evolve into small-scale plumes that rapidly coalesce into larger structures, resulting in turbulent convection throughout the lower layer. The turbulent convective velocity in our experiments increases approximately as the cube root of the rain buoyancy flux, implying little or no dependence on viscous and chemical diffusivities. Applying diffusion-free scaling laws for magnetic field generation, we find that precipitation-driven convection can be an effective dynamo mechanism in planetary cores provided the precipitation buoyancy flux is large and the convecting region is deep and nearly adiabatic.
\end{abstract}

Key words: Rain-driven convection, planetary dynamos, iron snow, magnesium precipitation, convection experiments, scaling laws

* Corresponding author: olson@jhu.edu

(C) 2016. This manuscript version is made available under the Elsevier user license http://www.elsevier.com/open-access/userlicense/1.0/ 


\section{${ }_{25} \quad 1 \quad$ Introduction}

26 Multi-phase flows involving liquids plus solids or several immiscible liquids have been pro-

27 posed as power sources for a number of planetary dynamos, both large and small (Breuer

28 et al., 2015). Examples include iron-snow precipitation in the iron alloy cores of Mercury

29 (Villim et al., 2010; Dumberry and Roldovini, 2015) and Ganymede (Hauck et al., 2006;

Rückriemen et al., 2015; Christensen, 2015), in terrestrial exoplanets (Gaidos et al., 2010), precipitation of low-density constituents near the top of Earth's iron-rich core for the early geodynamo (Buffett et al., 2000; O’Rourke and Stevenson, 2016; Badro et al., 2016), and helium rain in Saturn and other hydrogen-rich giant planets (Stevenson, 1980; Fortney and Hubbard, 2004).

Although the compositions of both the precipitate and the core fluid, and the ways envisioned to generate fluid motions differ among the planets and satellites, the underlying mechanics are fundamentally similar in each case, as Figure 1 illustrates. First, cooling of the planet leads to saturation of one or more components of the conducting fluid. Nucleation of that component produces liquid drops or solid grains, which precipitate as the equivalents of rain, sleet, graupel, or snow.

In Mercury and Ganymede, the usual assumption is that dense iron-snow precipitates downward through the upper, cooler portions of their molten iron alloy cores (Dumberry and Roldovini, 2015; Christensen, 2015). Falling into the deeper and warmer portions of the core, the iron-snow melts, increasing the density and destabilizing the fluid there. The core then consists of a two-layer system, with a precipitation-dominated region overlying a convectiondominated region, shown in Figure 1a. More complex layering is possible, including staircase structures, particularly if the melting curve and the temperature profile are irregular (Vilim 
et al., 2010; Dumberry and Roldovini, 2015), or if double diffusive processes occur. In general, however, it is usually concluded that the precipitating regions are stable and that dynamo action is concentrated in the convective region (Christensen and Wicht, 2008). The process is similar to convective turbulence in Earth's troposphere, when precipitation falling from clouds as virga snow sublimates before reaching the ground, cooling and destabilizing the air below the cloud base (Kudo, 2013).

A related but somewhat different precipitation scenario has been proposed for the Earth's core in the deep past. Evidence for a geomagnetic field of similar strength as the presentday field extends to 3.4 Ga (Tarduno et al., 2010) and possibly 4.2 Ga (Tarduno et al., 2015), whereas current estimates place the age of the inner core at $1 \mathrm{Ga}$ or less (Olson et al., 2015), highlighting the need for geodynamo energy sources other than inner core growth. It has been hypothesized that, as the core cooled from an initially high temperature state, nucleation of weakly soluble magnesium-bearing grains or drops occurred (O'Rourke and Stevenson, 2016; Badro et al., 2016). Positively buoyant in Earth's iron-rich core, the magnesium-bearing compounds precipitated upward and accumulated at the core-mantle boundary (CMB), as shown schematically in Figure 1b. Removal of magnesium left the residual core fluid denser and therefore unstable, enabling a compositional convective region to develop, thereby helping to maintain the early geodynamo.

Another example of precipitation-driven flow has been proposed for gas giant planets, Saturn in particular (Stevenson, 1980), but also giant exoplanets (Fortney and Hubbard, 2004). There, cooling produces supersaturated conditions for helium in the outer portion of the hydrogen-rich fluid envelope of the planet, a situation broadly similar to Figure 1a. It is hypothesized that helium rainfall stabilizes the density profile in a precipitation-dominated layer, allowing strong horizontal shear flows to develop. It has been shown that dynamo 
action driven by strong shear constrains the external magnetic field to be highly axisymmetric (Stanley, 2010; Cao et al., 2012), as observed on Saturn (Cao et al., 2011).

Thermochemical evolution calculations reveal that the rate of gravitational potential energy release in the saturation-precipitation process is proportional to the cooling rate of the fluid (Rückriemen et al., 2015). For plausible planetary cooling rates, the precipitation mechanisms described above are expected to release substantial amounts of gravitational potential energy per unit time, and therefore hold potential for dynamo action. Yet, little is known about the multi-phase flows involved in precipitation-driven convection, much less their ability to produce efficient planetary dynamos. In particular, there are questions about whether the potential energy released by precipitation converts efficiently to kinetic energy of fluid motion, and whether the kinetic energy is produced at scales that are large enough for dynamo action.

Precipitation may also play an important role during late-stage planetary accretion. It has been hypothesized that late-stage giant impacts fragment core-forming metals in a deep magma ocean (Tonks and Melosh, 1993; Nakajima and Stevenson, 2015) that might extend to the core (Labrosse et al., 2007). For some impacts, complete fragmentation could produce iron rainfall from the mantle directly into the core (Ichikawa et al., 2010; Deguen et al., 2014; Kraus et al., 2015). In this scenario, high-pressure, high-temperature metal-silicate interactions predict that the metallic rain absorbs large concentrations of lighter elements from the magma (Takafuji et al., 2005; Siebert et al., 2011) and would enter the core with a large density deficit, rather than a density excess, possibly contributing to the stable layering inferred at the top of the present-day outer core (Helffrich and Kaneshima, 2010; Landeau et al., 2016). 
In this study we investigate these issues using analog laboratory experiments of raindriven convection. We exploit differences in interfacial tension to create a transition from a precipitation-dominated region to a convection-dominated region in a two-layer fluid with geometry shown in Figure 1c. In both the laboratory and in nature, precipitation-driven convection is intrinsically non-uniform on multiple scales. On the smallest scales, it is granular in space and time because it originates from the dissolution of individual drops or particles. It is also heterogeneous at intermediate time and length scales, due to variability in the local precipitation rate, which in our experiments comes from random fluctuations in the rain production apparatus in Figure 1c, and in natural systems comes from lateral heterogeneity in temperature, composition, and the velocity of the fluid through which the precipitation falls. A fundamental assumption is that these small and intermediate scale heterogeneities average out, so that on larger scales the precipitation induces a constant and horizontally uniform buoyancy flux, analogous to turbulent Rayleigh-Bénard convection with fixed heat flux boundary conditions (Verzicco and Sreenivasan, 2008; Johnston and Doering, 2009; Huang et al., 2015). However, because precipitation-driven convection has received so little attention, the validity of this assumption as applied to planetary dynamos is an open question.

\section{Rain-driven convection experiments}

Figures 2-5 show results of laboratory experiments in which a two-layer fluid is subject to a uniform intensity rainfall of a third fluid. The rain is a dyed aqueous solution, immiscible in the upper fluid, a low-density, low-viscosity silicone oil, but miscible in the lower fluid, pure water in these experiments. The two fluids are confined in a rectangular plexiglass tank with a square $25 \times 25 \mathrm{~cm}$ cross-sectional area shown in Figure 1c, into which a $25 \mathrm{~cm}$ diameter 
circular shower head is fitted, consisting of 100 equally-spaced, $0.75 \mathrm{~mm}$ diameter spigots connected via a flow meter and control valve to a head tank containing the rain fluid. With this setup, the corner regions of the tank receive less direct precipitation compared to the central region. In our experiments, we concentrate on the action in the central region, where the rain is nearly uniform. Back lighting with a rectangular diode array is used to illuminate the tank.

We consider two types of rain, distinguished by their density relative to the lower fluid. In high-density rain experiments, hereafter referred to as Type 1 experiments, the rain fluid consists of a water- $\mathrm{NaCl}$ solution with organic dye, the salt and dye concentrations adjusted to produce the desired density excess with respect to the lower fluid. In low-density rain experiments, referred to as Type 2, the rain fluid consists of a water-ethanol solution with organic dye, their concentrations adjusted to produce the desired density deficit with respect to the lower fluid. Properties of the experimental fluids and a comparison of the dimensionless parameters in the experiments and in planetary cores are given in Table 1.

In the high-density Type 1 rain experiment shown in close-up in Figures 2a and 2b, the rain has an initial density excess of $\Delta \rho / \rho_{L}=+1.0 \%$ with respect to the lower fluid, the average rainfall intensity is $R=4.5 \times 10^{-4} \mathrm{~m} \mathrm{~s}^{-1}$ (approximately $28 \mathrm{ml} \mathrm{s}^{-1}$ total rain rate) applied over approximately 120 s. The corresponding rain buoyancy flux, defined as (see Appendix)

$$
F=\frac{g \Delta \rho}{\rho_{L}} R
$$

where $g$ is gravity, is $F=4.5 \times 10^{-5} \mathrm{~m}^{2} \mathrm{~s}^{-3}$ in this experiment. In the low-density Type 2 rain experiment shown in close-up in Figures 2c and 2d, the rain density deficit is $-1.0 \%$ and the average rainfall intensity is the same as in the accompanying Type 1 experiment, so that the rain buoyancy flux in that case is $F=-4.5 \times 10^{-5} \mathrm{~m}^{2} \mathrm{~s}^{-3}$. 
In both Type 1 and 2 experiments, rain precipitates through the upper layer and accumulates as a binary emulsion at the interface with the lower fluid. The drops fall along nearly vertical trajectories in the upper layer. We do not observe substantial lateral deflections or clustering of the drops as they fall, even in the highest rain intensity experiments, as would be the case if the rainfall generated coherent large-scale motions in the upper layer. We emphasize this point because it relates to the question of the capacity for dynamo action in precipitation-dominated regions of planetary cores.

Gravitational separation of the fallen rain and the upper layer fluid occurs within the two-phase emulsion zone shown in Figure 2 for both Type 1 and Type 2 experiments. The various steps in the gravitational separation processes within the emulsion are the same as described in detail by Sato and Sumita (2007) and Gilet et al., (2007) for the case in which the negatively buoyant component is discontinuous (localized in drops) and the neutral component forms the connected pore network. Drop dissolution ejects small volumes of the rain fluid downward, forming millimeter-sized ring vortices below the emulsion interface, while the less dense silicone oil percolates back into the upper layer.

The subsequent behavior of the rain fluid injected into the lower layer depends on its density relative to the lower fluid. In the case of excess density rain in Type 1 experiments, the vortex rings formed by drop dissolution evolve into small-scale, negatively buoyant compositional plumes, as shown in Figure 2a. The time sequence in Figures 2a and 2b shows how these small-scale compositional plumes coalesce into larger plumes that drive a still larger-scale turbulent convective overturn, mixing and homogenizing the entire lower fluid. In contrast, in Type 2 experiments with density-deficient rain, the small-scale compositional plumes are positively buoyant and barely penetrate into the lower fluid, as shown in Figures $2 \mathrm{c}$ and $2 \mathrm{~d}$. Instead of producing turbulence throughout the lower layer, the rain products 
accumulate below the emulsion interface, producing a stratified rain product layer that grows with time.

The intensity of convection in the lower layer in Type 1 experiments is governed by a flux Rayleigh number defined in terms of $F$ (see Appendix)

$$
R a_{F}=\frac{F d_{L}^{4}}{\nu^{3}}
$$

where $d_{L}$ and $\nu$ are the initial lower fluid depth and kinematic viscosity, respectively. For the Type 1 experiment shown in Figures $2 \mathrm{a}$ and $2 \mathrm{~b}$, using the lower fluid depth $d_{L}$ given in Table 1, the flux Rayleigh number is $R a_{F}=4.5 \times 10^{10}$. We measure the intensity of the convective turbulence in terms of a Reynolds number based on the lower fluid depth and the average velocity of the plumes $w$ (see Appendix)

$$
R e=\frac{w d_{L}}{\nu},
$$

4 Th

175 ave

ent shown in Figures $2 \mathrm{a}$ and $2 \mathrm{~b}, R e \simeq 1.8 \times 10^{3}$.

The image sequence in Figure 3 shows large-scale views of the development of the convecting region in an $0.1 \%$ excess density (Type 1 ) rain experiment at $R a_{F}=4.5 \times 10^{9}$. The emergence of large-scale flows through plume growth and coalescence is evident in this sequence. At $9 \mathrm{~s}$ after rain injection begins (Figure 3a) the small size of the plumes corresponds to the scale at which vorticity is injected into the lower layer following the coalescence of individual rain drops. At $24 \mathrm{~s}$ after the initial injection (Figure 3b), clusters of the small plumes have merged to form larger plumes. Plume mergers continue as the mixing layer and the large-scale circulation approach the base of the lower layer at $39 \mathrm{~s}$ (Figure 3c). The final 
image Figure 3d shows nearly complete homogenization on the lower layer by the overturning circulation. The progressive upward displacement of the emulsion at the rain-convection interface in Figures 3a-d is due to filling of the lower layer by the rain fluid.

The two images in Figure 4 compare Type 1 rain-driven turbulent convection with large and small density excess, respectively. In Figure 4a, the rain density excess is $0.03 \%$, the flux Rayleigh number is $R a_{F}=1.35 \times 10^{9}$ and the plume Reynolds number is $R e=605$, whereas in Figure 4b the rain density excess is $2.5 \%$, the flux Rayleigh number is approximately $R a_{F}=1.13 \times 10^{11}$, nearly two orders of magnitude larger, and the plume Reynolds number is approximately $R e=2500$. Note the increase in turbulent mixing from the higher density rainfall and the shorter growth time of the plumes in Figure 4b compared to Figure 4a. But in terms of their large-scale structure, the two flows are remarkably similar, despite the nearly two orders of magnitude difference in $R a_{F}$.

Figure 5 shows the relationship between the plume Reynolds number defined in (3) and the flux Rayleigh number, from five different Type 1 rain convection experiments. Figure 5 also shows a power-law fit to the data of the form

$$
R e=c R a_{F}^{\beta}
$$

in which $c=0.17 \pm 0.02$ and $\beta=0.32 \pm 0.02$. Within experimental error, (4) is indistinguishable from the $\beta=1 / 3$-power law exponent that corresponds to ideal inertial scaling (see Appendix). In terms of dimensional variables, a 1/3-power law relationship between Re and $R a_{F}$ implies $w \propto\left(F d_{L}\right)^{1 / 3}$, i.e., the plume velocity is independent of the viscosity and the diffusivity, and depends only on the buoyancy flux and the convective layer depth. In our experiments, we mostly varied the buoyancy flux, by independently varying the rain density and the precipitation rate. In contrast, the lower layer depth was varied only by 
small amounts, so the implied $d_{L}^{1 / 3}$-dependence of $w$ was not actually tested.

We can compare our plume Reynolds number scaling with the plume Reynolds numbers measured in thermal convection experiments of the classical Rayleigh-Bénard type, in which the top and bottom boundaries are rigid and isothermal. Figure 5 shows a semi-theoretic scaling law for the plume Reynolds number derived by Grossmann and Lohse (2002), which has the form

$$
R a_{F}=(N u-1) P r^{-2} R a=c_{1} \frac{R e^{2}}{f(R e)}+c_{2} R e^{3},
$$

in which $R a$ and $N u$ are the Rayleigh number and the Nusselt number in Rayleigh-Bénard convection defined in terms of the temperature difference across the fluid (see the Appendix), where $\operatorname{Pr}=\nu / \kappa$ is the Prandtl number ( $\kappa$ being thermal diffusivity), $f(x)=\left(1+x^{2}\right)^{1 / 4} / x^{1 / 2}$, and $c_{1}$ and $c_{2}$ are empirical coefficients that depend on the experimental geometry and boundary conditions (Grossmann and Lohse, 2003), and also on the technique used to measure the plume velocities (Ahlers et al., 2009).

Fitting (5) to turbulent Rayleigh-Bénard convection experiments in a variety of fluids, Grossmann and Lohse (2002) obtain $c_{1} \simeq 8.7$ and $c_{2} \simeq 1.45$. The resulting scaling law is indicated by the dashed line in Figure 5. A similar scaling has been found to hold for Rayleigh-Bénard convection in cylindrical domains, but with some variations in the coefficients (Ahlers and Xu, 2001; Funfschilling et al., 2005). In Figure 5 we indicate the sensitivity to geometry and boundary conditions with the background gray shading. We note that the trend of our data is hardly distinguishable from (5), and the offset is only about 40\%, an amount which can be expected in light of the differences in boundary conditions, geometry, and the fact that we measure the overall plume travel time in our rain convection experiments versus mid-depth plume travel times in the conventional Rayleigh-Bénard convection experiments. 


\section{${ }_{231} 3$ Implications for precipitation-driven dynamos}

${ }_{232}$ We quantify the dynamo capacity of precipitation-driven convection in planetary core using scaling laws for the fluid velocity and the magnetic field intensity generated by thermochemical convection obtained from numerical dynamo systematics (Christensen and Aubert, 2006; Aubert et al. 2009). These scaling laws include the effects of spherical shape, selfgravitation, and planetary rotation, which are missing from our experiments, and assume that the fluid velocity and the magnetic induction are independent of diffusive effects, including the viscosity. The fact that our experimental convective velocities are consistent with inertial (i.e., viscosity-independent) scaling provides some justification for using such diffusion-free dynamo scaling laws in this application.

We assume that the planet's core is divided into a outer precipitation-dominated region and a central convection-dominated region, separated at a radius $r_{c}$. We can write the precipitation buoyancy flux at the top of the convective region as (1), where now $g$ is gravity at radius $r_{c}, \rho_{L}$ is the convecting region density, $\Delta \rho$ is the density difference between the convecting region and the precipitation, and $R$ is the rate of precipitation, solid or liquid, evaluated at the top of the convecting region. Magnetic field generation depends on $F$ through the magnetic Reynolds number of the convection $R m$. In an entirely molten convecting sphere with radius $r_{c}$,

$$
R m=\frac{u r_{c}}{\eta}
$$

where $\eta$ is the magnetic diffusivity of the fluid and $u$ is the rms fluid velocity. For full-sphere geometries and assuming an adiabatic heat flux at the CMB, dynamo scaling relationships (Aubert et al., 2009) yield

$$
R m \simeq 1.3 p^{0.42} P m E^{-1}
$$


where $P m=\nu / \eta$ is the magnetic Prandtl number, $E=\nu / \Omega r_{c}^{2}$ is the Ekman number,

$$
p=\frac{3 F}{5 \Omega^{3} r_{c}^{2}}
$$

is the convective power, and $\Omega$ is angular velocity of planetary rotation. The critical magnetic Reynolds number for convective dynamo onset is $R m_{\text {crit }} \simeq 40$ (Christensen et al., 1999).

We first consider magnesium precipitation in Earth's core prior to inner core nucleation, recently proposed by O'Rourke and Stevenson (2016) and later considered by Badro et al. (2016). Rising precipitation in the form of buoyant magnesium oxides and silicates would leave behind a dense iron-rich residual fluid, promoting compositional convection deeper in the core. If we assume that the background state of the core is well mixed (i.e., adiabatic), then very low intensity precipitation of this type could initiate the geodynamo. According to (1), (7), (8) and the data in Table 2, a precipitation rate of just a few $\mathrm{m} \mathrm{Gyr}^{-1}$ produces $R m_{\text {crit }}$ under these conditions.

More intense magnesium precipitation is needed to maintain a finite intensity magnetic field, even if the background state of the core is well mixed. According to dynamo model systematics (Olson and Christensen, 2006) the dipole magnetic moment $\mathcal{M}$ in the dipoledominated regime depends on the buoyancy flux from precipitation $F$ approximately as

$$
\mathcal{M} \simeq 1.8\left(\frac{\rho}{\mu_{0}}\right)^{1 / 2} r_{c}^{10 / 3} F^{1 / 3},
$$

where $\mu_{0}$ is free-space magnetic permeability. In order to sustain a magnetic field with dipole moment $\mathcal{M}=50 \mathrm{ZAm}^{2}$, comparable to the time-averaged Proterozoic geomagnetic field intensity (Biggin et al., 2015), (9) along with the data in Table 2 predict that a precipitation rate of $R \simeq 400 \mathrm{~m} \mathrm{Gyr}^{-1}$ is required. This is a negligibly small magnesium addition to the mantle and would not materially affect geochemical tracers such as Hf-W isotopic ratios (Rudge et al., 2010) or mantle siderophiles (Chabot et al., 2005). It would slightly increase Earth's 
rotation rate, but by less than $1 \%$, far too small to counteract the angular deceleration from tidal braking (Denis et al., 2011).

Far more intense precipitation would be needed for dynamo action if the core was stratified, either thermally or by composition. If the heat loss from the convective region is less than the heat conducted down the core adiabat, a plausible situation in view of the high thermal conductivity of the core (Gomi et al., 2013; Labrosse, 2015), then an additional compositional buoyancy flux must be added to balance the negative thermal buoyancy flux due to the subadiabatic thermal gradient, in order to homogenize the core fluid. In terms of the total heat loss from the convecting region $Q$, the additional thermal buoyancy flux is given by $F_{T}=\left(Q-Q_{a d}\right) / \rho A_{c} H_{c}$, where $Q_{a d}$ is the adiabatic heat loss, $A_{c}=4 \pi r_{c}^{2}$ is the surface area of the convecting region, and $H_{c}$ is its temperature scale height. The net buoyancy flux that enters into the expression for the convective power (8) then becomes $F+F_{T}$. If the thermal buoyancy flux $F_{T}$ is negative because $Q<Q_{a d}$, then a larger rain-produced buoyancy flux $F$ is required in order to compensate for the subadiabatic heat loss.

The effects of core heat flux and magnesium precipitation rate on the dynamo structure and the induced dipole moment are shown in Figure 6, where we have assumed a convecting region outer radius of $r_{c}=3480 \mathrm{~km}$, equal to the present-day CMB radius, and an adiabatic heat flux of $0.1 \mathrm{~W} \mathrm{~m}^{-2}$, equivalent to $Q_{a d} \simeq 15 \mathrm{TW}$. Line contours indicate the induced dipole moment in $\mathrm{ZAm}^{2}$ from (9) and the local Rossby number $R o_{\ell}$, which has been proposed as a control parameter for the transition from dipolar to multipolar dynamo states (Christensen and Aubert, 2006; Aubert et al., 2009):

$$
R o_{\ell} \simeq 0.54 p^{0.48} E^{-0.32}(\operatorname{Pr} P m)^{0.19}
$$

294 The dipolar-multipolar transition occurs near $R_{\ell} \sim 0.1$, according to many previous studies 
(Christensen and Aubert, 2006; Aubert et al., 2009; Oruba and Dormy, 2014). Shadings in Figure 6 indicate dynamo regimes that are subcritical (labeled no-dynamo), convection driven by precipitation against stable thermal stratification (labeled precipitation dynamo), and convection driven by precipitation acting in concert with unstable thermal stratification (labeled thermo-precipitation dynamo).

Magnesium precipitation can maintain a strong dipolar dynamo even under subadiabatic conditions, provided the precipitation rate is high enough. Suppose, for example, that $Q=12$ TW, equivalent to $q_{c m b} \simeq 0.08 \mathrm{~W} \mathrm{~m}^{-2}$ for the average local heat flux at the CMB. To maintain a dipole moment of $50 \mathrm{ZAm}^{2}$ with this core heat flux, Figure 6 indicates that a magnesium rain rate of approximately $R=2.8 \mathrm{~km} \mathrm{Gyr}^{-1}$ is required. For purposes of comparison, this is approximately the same as the present-day mean rate of sediment accumulation on the Pacific ocean floor (Olson et al., 2016), a modern analog that demonstrates how sparse this type of precipitation would be in the Earth's core.

With these properties, $R o_{\ell}<0.1$, indicating the dynamo would be dipolar, consistent with most Proterozoic paleomagnetic data (Biggin et al., 2015). However, a precipitation dynamo with these parameters lies perilously close to the subcritical regime in Figure 6, such that a modest reduction in core heat flux would cause it to fail. The situation is made worse by the fact that the precipitation rate $R$ is coupled to $Q$ through the cooling rate of the core, so that a reduction in $Q$ also reduces $R$, further increasing the chances of dynamo failure.

The results in Figure 6 assume the convective region occupies the entire Earth's core. In other planets the convective region may occupy only a fraction of the core, and if so, the precipitation rate required for dynamo action can be very high. For example, Figure 7 
shows contours of magnetic Reynolds number from (7) and local Rossby number from (10) as functions of convecting radius and iron-snow precipitation rate for Mercury, based on the parameter values in Table 2 and assuming a solid inner core is absent or negligibly small. If the convective radius exceeds $200 \mathrm{~km}$, a minimum precipitation rate near $30 \mathrm{~km} \mathrm{Gyr}^{-1}$ is needed for dynamo onset, but in a smaller convecting region, with $r_{c}<100 \mathrm{~km}$, say, dynamo action would require far higher precipitation rates.

Another implication of Figure 7 is that iron-snow precipitation can generate high magnetic Reynolds number conditions in a deep convective region in Mercury, but the large values of $R o_{\ell}$ imply that this convection would induce a multipolar field, rather than a dipole dominant field. Accordingly, the scaling relation (9) does not apply here. Our prediction of a multipolar dynamo field is seemingly in contradiction with the offset dipolar field observed in orbit around Mercury (Anderson et al., 2011). It is, however, consistent with a proposed deep dynamo mechanism for Mercury by Christensen (2006), which postulates that the non-dipolar components of the dynamo magnetic field generated deep within the Mercury's core are electromagnetically screened in an overlying stable layer. According to our experiments, such electromagnetic screening might occur in the precipitation-dominated layer because it lacks large-scale circulation.

Figure 8 shows the dynamo regimes for Ganymede, calculated according to the same scaling laws and assumptions as used for Earth and Mercury but with the property values for Ganymede in Table 2. The model for Ganymede differs from Mercury in having a smaller convecting region and a lower assumed thermal conductivity. A convective dynamo driven by iron snow precipitation, possibly aided by an unstable thermal gradient, is predicted for most precipitation rates. The contours show the local Rossby number $R o_{\ell}$. The range includes the dynamo configurations described by Christensen (2015): a dipole-dominated 
dynamo at low $R_{o_{\ell}}$ and multipolar dynamo at high $R_{\ell}$.

\section{Conclusions}

In this paper we describe a new class of convective flows, driven by buoyancy acquired through precipitation. This form of convection has been proposed for evolving planetary cores, where cooling results in nucleation, precipitation, and dissolution of buoyant constituents. Our rain-driven experiments are analogous to precipitation-driven systems in planetary interiors in several respects, although there are important differences.

Similarities include (1) the basic two-layer dynamical structure, with a precipitation region separated from a region where the precipitation is dissolved, with the possibility of compositional convection; (2) a thin multi-component transition zone separating these regions where the buoyancy for convection originates; (3) buoyancy production on the scale of the individual precipitates, followed by plume coalescence.

Significant differences include, for the experiments (1) lack of phase changes (solidification and melting) and latent heat exchange, which could contribute to the convection; (2) absence of rotational and magnetic field effects, which tend to stabilize the convection; (3) the idealized plane-layer geometry, which ignores the changes in fluid volume and gravity with radius; and (4) double-diffusive convection, which could increase the dynamo capacity of this system. Each of these merit additional study.

In addition, there are internal structure differences between our experiments and some of the scenarios envisioned for planetary interiors. In Earth's core, for example, it is supposed that extraction of upward precipitating magnesium or other light elements would yield a negatively buoyant residual liquid, whereas in our experiments, the negatively buoyant liquid 
is produced through addition of downward precipitation. In cores dominated by the Fe-FeS system, the geometry might be inverse of our experiment. For example, for core compositions on the FeS side of the eutectic, convection might be due to sulfur-rich fluid rising from a deep iron snow zone (Hauck et al. 2006; Chen et al. 2008). In spite of these differences, we claim that the fundamental effects of precipitation on the dynamics of these systems are captured in our experiments.

A key finding of our study is the process by which stochastic precipitation self-organizes to generate larger-scale convective motions. In our experiments, this organization begins during gravitational separation within multi-component emulsion layers, whereas in planetary interiors it is generally hypothesized to occur near the pressure-temperature horizon that corresponds to a phase transition. In both situations, however, the fluid buoyancy develops on the scale of the isolated particles or drops, generating micro-scale plumes that must coalesce in order to form the larger plume structures that induce convective overturn.

Although substantial gravitational potential energy may be released in the precipitationdominated region of a planetary core, this does not guarantee that large-scale magnetic induction occurs there. If the only fluid motions in the precipitation zones are in particle wakes, which have lateral dimensions on the scale of the particles themselves, these will be quickly dissipated by viscous or Lorentz forces, and will contribute little (if anything) to dynamo action. In contrast, our experiments show that the convective regions have far more capacity for dynamo action. In planetary cores, the rate of precipitation needed to maintain a convective dynamo is not excessive, particularly if the precipitation is intense and strongly buoyant, and if the convecting region is deep.

Lastly, we note that previous studies have found that diffusive boundary layer effects, 
387

388

389

390

391

392

393

394

395

396

including viscosity, remain controlling factors in heat transfer in Rayleigh-Bénard convection when planetary rotation is included, even at high Rayleigh numbers (King et al., 2009,

Stellmach et al., 2014). It would be revealing, therefore, to determine how the velocity scaling for precipitation-driven convection changes when rotation is added.

Acknowledgements: We gratefully acknowledge support from grant EAR-0909622 and FESD grant EAR-1135382 from the National Science Foundation. This paper benefited from helpful comments by two anonymous referees.

Author contributions: BH, ML, and PO jointly conducted the experiments; PO and ML jointly made the calculations; PO and ML prepared the figures, tables, and references; PO and ML jointly wrote the paper. 


\section{Appendix}

where $\rho$ is the mean density, $\mathbf{u}$ is the fluid velocity, $\nu$ is kinematic viscosity, and

$$
F=\rho^{\prime}(\mathbf{g} \cdot \mathbf{u}) / \rho
$$

403 is the buoyancy flux, in which $\mathbf{g}$ is the vector gravity and $\rho^{\prime}$ is the density perturbation, 413 asterisks) as

$$
\frac{w^{3}}{2 d} \nabla^{*} \cdot\left(u^{* 2} \mathbf{u}^{*}\right)=F
$$

${ }_{414}$ where. In order for this force balance to hold, the convective velocity must scale like

$$
w \sim(F d)^{1 / 3},
$$


415 as given in the text.

${ }_{416}$ Rayleigh-Bénard thermal convection experiments use a different Rayleigh number, de417 fined as

$$
R a=\alpha g \Delta T d^{3} / \kappa \nu
$$

${ }_{418}$ where $\alpha$ and $\kappa$ are thermal expansion and thermal diffusivity, respectively, and $\Delta T$ is the 419 temperature difference across the fluid. Heat transfer in Rayleigh-Bénard convection is 420 usually measured in terms of the Nusselt number, defined as

$$
N u=q d / k \Delta T
$$

${ }_{421}$ where $q$ and $k$ are heat flux (conventionally measured on the fluid layer boundary) and ${ }_{422}$ thermal conductivity of the fluid, respectively. The definitions (16) and (17) are used in the 423 r.h.s. of equation (5). 
Ahlers, G., Xu, X., 2001. Prandtl-number dependence of heat transport in turbulent Rayleigh-Bénard convection. Phys. Rev. Lett. 86, 3320-3323.

Ahlers, G., Grossmann, S., Lohse, D., 2009. Heat transfer and large scale dynamics in turbulent Rayleigh-Bénard convection. Rev. Mod. Phys. 81(2), 503-537.

Anderson, B. J., et al., 2011. The global magnetic field of Mercury from MESSENGER orbital observations. Science 333.6051, 1859-1862.

Aubert, J., Labrosse, S., Poitou, C., 2009. Modelling the paleo-evolution of the geodynamo. Geophys. J. Int. 179, 1414-1428.

Badro, J., Siebert, J., Nimmo, F., 2016. An early geodynamo driven by exsolution of mantle components from Earths core. Nature doi:10.1038/nature18594.

Biggin, A., Piispa, E., Pesonen, L., Holme, R., Paterson, G., Veikkolainen, T., Tauxe, L., 2015. Palaeomagnetic field intensity variations suggest Mesoproterozoic inner-core nucleation. Nature 526 (7572), 245-248.

Breuer, D., Rueckriemen, T., Spohn, T., 2015. Iron snow, crystal floats, and inner-core growth: modes of core solidification and implications for dynamos in terrestrial planets and moons. Prog. Earth Planet. Sci. 2(1), 1.

Buffett, B.A., Garnero, E.J., Jeanloz, R., 2000. Sediments at the top of the core. Science 290, 1338-1342.

Cao, H., Russell, C. T., Christensen, U. R., Dougherty, M. K., Burton, M. E., 2011. Saturn's very axisymmetric magnetic field: No detectable secular variation or tilt. Earth Planet. 
Sci. Lett. 304(1), 22-28.

Cao, H., Russell, C. T., Wicht, J., Christensen, U. R., Dougherty, M. K., 2012. Saturns high degree magnetic moments: Evidence for a unique planetary dynamo. Icarus 221(1), 388-394.

Chabot, N. L., Draper, D. S., Agee, C. B., 2005. Conditions of core formation in the Earth: constraints from nickel and cobalt partitioning. Geochim. Cosmochim. Acta 69, 2141-2151.

Chen, B., Li, J., Hauck, S.A., 2008. Non-ideal liquidus curve in the FeS system and Mercurys snowing core. Geophys. Res. Lett. 35, L07201.

Christensen, U.R., 2006. A deep dynamo generating Mercury's magnetic field. Nature 444, 1056-1058.

Christensen, U. R., 2015. Iron snow dynamo models for Ganymede. Icarus 247, 248-259.

Christensen, U. R., Olson, P., Glatzmaier, G. A., 1999. Numerical modelling of the geodynamo: a systematic parameter study. Geophys. J. Int. 138, 393-409.

Christensen, U. R., Aubert, J., 2006. Scaling properties of convection-driven dynamos in rotating spherical shells and application to planetary magnetic fields. Geophys. J. Int. $166,97-114$.

Christensen, U.R., Wicht, J., 2008. Models of magnetic field generation in partly stable planetary cores: Applications to Mercury and Saturn. Icarus 196, 16-34.

Deguen, R., Landeau, M., Olson, P., 2014. Turbulent metal-silicate mixing, fragmentation, and equilibration in magma oceans. Earth Planet. Sci. Lett. 391, 274-287. 
Deng, L., Deagle, C., Fei, Y., Shahar, A., 2013. High pressure and temperature electrical resistivity of iron and implications for planetary cores. Geophys. Res. Lett. 40, 3337.

Denis, C., Rybicki, K. R., Schreider, A. A., TomeckaSucho, S., Varga, P., 2011. Length of the day and evolution of the Earth's core in the geological past. Astronomische Nachrichten 332(1), 24-35.

Dumberry, M., Rivoldini, A., 2015. Mercury's inner core size and core-crystallization regime. Icarus 248, 254-268.

Dziewonski, A. M., Anderson, D. L., 1981. Preliminary reference Earth model. Phys. Earth Planet. Inter. 25, 297-356.

Fortney, J.J., Hubbard, W.B., 2004. Effects of helium phase separation on the evolution of extrasolar giant planets. Astrophys. J. 608, 1039-1049.

Funfschilling, D., Brown, E., Nikolaenko, A., Ahlers, G., 2005. Heat transport by turbulent Rayleigh-Bénard convection in cylindrical cells with aspect ratio one and larger. J. Fluid Mech. 536, 145-154.

Gaidos, E., Conrad, C. P., Manga, M., Hernlund, J., 2010. Thermodynamic constraints on rocky exoplanet dynamos. Astrophys. J. 718, 596-609.

Gilet, T., Mulleners, K., Lecomte, J. P., Vandewalle, N., Dorbolo, S., 2007. Critical parameters for the partial coalescence of a droplet. Phys. Rev. E 75, 036303.

Gomi, H., Ohta, K., Hirose, K., Labrosse, S., Caracas, R., Verstraete, M. J., Hernlund, J. W., 2013. The high conductivity of iron and thermal evolution of the Earth's core. Phys. Earth Planet. Inter. 224, 88-103. 
Grossmann, S., Lohse, D., 2002. Prandtl and Rayleigh number dependence of the Reynolds number in turbulent thermal convection. Phys. Rev. E 66, 016305.

Grossmann, S., Lohse, D., 2003. On geometry effects in Rayleigh-Bénard convection. J. Fluid Mech. 486, 105-114.

Hauck, S. A., Aurnou, J. M., Dombard, A. J., 2006. Sulfur impact on core evolution and magnetic field generation on Ganymede. J. Geophys. Res. 111, E09008.

Helffrich, G., Kaneshima, S., 2010. Outer-core compositional stratification from observed core wave speed profiles. Nature 468, 807-809.

Hirose, K., Labrosse, S., Hernlund, J., 2013. Composition and State of the Core. Ann. Rev. Earth Planet. Sci. 41, 657-691.

Huang, S.-D., Wang, F., Xi, H.-D., Xia, K.-Q., 2015. Comparative experimental study of fixed temperature and fixed heat flux boundary conditions in turbulent thermal convection. Phys. Rev. Lett. 115, 15452.

Ichikawa, H., Labrosse, S. Kurita, K., 2010. Direct numerical simulation of an iron rain in 221 the magma ocean. J. Geophys. Res. 115, doi:10.1029/2009JB006427.

Johnston, H., Doering, C.R., 2009. Comparison of turbulent thermal convection between conditions of constant temperature and constant flux. Phys. Rev. Lett. 102, 064501.

King, E. M., Stellmach, S., Noir, J., Hansen, U., Aurnou, J. M., 2009. Boundary layer control of rotating convection systems. Nature, 457(7227) 301-304.

Kraus, R. G., Root, S., Lemke, R. W., Stewart, S. T., Jacobsen, S. B., Mattsson, T. R., 2015. Impact vaporization of planetesimal cores in the late stages of planet formation. 
Nature Geoscience, 8(4), 269-272.

Kudo, A., 2013. The generation of turbulence below midlevel cloud bases: The effect of cooling due to sublimation of snow. J. Applied Meteor. Climat., 52(4), 819-833.

Labrosse, S., 2015. Thermal evolution of the core with a high thermal conductivity, Phys. Earth Planet Inter. 247, 36-55.

Labrosse, S., Hernlund, J. W., Coltice, N., 2007. A crystallizing dense magma ocean at the base of the Earth's mantle. Nature 450, 866-869.

Landeau, M., Olson, P., Deguen, R., Hirsh, B., 2016. Core merging and stratification following giant impact, Nature Geoscience DOI: 10.1038/NGEO2808.

Nakajima, M., Stevenson, D. J., 2015. Melting and mixing states of the Earth's mantle after the Moon-forming impact. Earth Planet. Sci. Lett. 427, 286-295.

Olson, P., Christensen, U. R., 2006. Dipole Moment Scaling for Convection-Driven Planetary Dynamos. Earth Planet. Sci. Lett. 250, 561-571.

Olson, P., Deguen, R. Rudolph, M.L., Zhong, S., 2015. Core evolution driven by mantle global circulation. Phys. Earth Planet. Inter., 243, 44-55.

Olson, P., Reynolds, E., Hinnov, L., Goswami, A., 2016. Variation of ocean sediment thickness with crustal age. Geochem. Geophys. Geosyst. 17, doi:10.1002/2015GC006143.

Poirier, J.-P., 2000. Introduction to the physics of the Earth's interior, 2nd Edition. Cambridge University Press.

O'Rourke, J. G., Stevenson, D.J., 2016. Powering Earth?s dynamo with magnesium precipitation from the core. Nature 529(7586), 387-389. 
Oruba, L., Dormy, E., 2014. Predictive scaling laws for spherical rotating dynamos. Geophys. J. Inter. 198(2), 828-847.

Rückriemen, T., Breuer, D., Spohn, T., 2014. The Fe snow regime in Ganymede's core: A deep-seated dynamo below a stable snow zone. J. Geophys. Res. Planets. 120(6), 1095-1118.

Rudge, J. F., Kleine, T., Bourdon, B., 2010. Broad bounds on Earth's accretion and core formation constrained by geochemical models. Nature Geoscience 3(6), 439-443.

Sato, M., Sumita, I., 2007. Experiments on gravitational phase separation of binary immiscible fluids. J. Fluid Mech. 591, 289-319.

Siebert, J., Corgne, A., Ryerson, F. J., 2011. Systematics of metal-silicate partitioning for many siderophile elements applied to Earth's core formation. Geochim. Cosmochim. Acta 75, 1451-1489.

Stanley, S., 2010. A dynamo model for axisymmetrizing Saturn's magnetic field. Geophys. Res. Lett. 37, L05201.

Stellmach, S., Lischper, M., Julien, K., Vasil, G., Cheng, J. S., Ribeiro, A., Aurnou, J. M., 2014. Approaching the asymptotic regime of rapidly rotating convection: Boundary layers versus interior dynamics. Physical review letters 113(25), 254501.

Stevenson, D.J., 1980. Saturns luminosity and magnetism. Science 208, 746-748.

Takafuji, N., Hirose, K., Mitome, M. Bando, Y., 2005. Solubilities of O and Si in liquid iron in equilibrium with $(\mathrm{Mg}, \mathrm{Fe}) \mathrm{SiO} 3$ perovskite and the light elements in the core. Geophys. Res. Lett. 32, L06313. 
Tarduno, J. A., Cottrell, R. D., Davis, W, J., Nimmo, F., Bono, R.K., 2015. A Hadean to Paleoarchean geodynamo recorded by single zircon crystals. Science 349(6247), $521-524$.

Tonks, W. B., H.J. Melosh, 1993. Magma ocean formation due to giant impacts. J. Geophys. Res. Planets 98(E3), 5319-5333.

Verzicco, R., Sreenivasan, K.R., 2008. Comparison of turbulent thermal convectin between conditions of constant temperature and constant flux. J. Fluid Mech. 595, 203-219.

Vilim, R., Stanley, S., Hauck II, S. A., 2010. Iron snow zones as a mechanism for generating Mercury's weak observed magnetic field. J. Geophys. Res. 115, E11003.

Vocaldo, L., Alfe, D., Gillan, M. J., Price, G. D., 2003. The properties of iron under core conditions from frst principles calculations. Phys. Earth Planet. Inter. 140, 101-125.

Ward, W. R., Colombo, G., Franklin, F. A., 1976. Secular resonance, solar spin down, and the orbit of Mercury. Icarus, 28(4), 441-452.

Williams, G. E., 2000. Geological constraints on the Precambrian history of Earth's rotation and the Moon's orbit. Rev. Geophys. 38, 37-59. 
Table 1: Table 1: Rain-Driven Convection Experiment Properties

\begin{tabular}{ccccc}
\hline Property & Notation & Units & $\begin{array}{c}\text { Experiment } \\
\text { Type } 1\end{array}$ & $\begin{array}{c}\text { Experiment } \\
\text { Type } 2\end{array}$ \\
\hline upper fluid density & $\rho_{U}$ & $\mathrm{~kg} \mathrm{~m}^{-3}$ & $0.82 \times 10^{3}$ & $0.82 \times 10^{3}$ \\
lower fluid density & $\rho_{L}$ & $\mathrm{~kg} \mathrm{~m}^{-3}$ & $1.0 \times 10^{3}$ & $1.0 \times 10^{3}$ \\
rain-lower fluid density difference & $\Delta \rho$ & $\mathrm{kg} \mathrm{m}^{-3}$ & $0.3-25$ & -10 \\
rain viscosity & $\nu_{r}$ & $\mathrm{~m}^{2} \mathrm{~s}^{-1}$ & $1 \times 10^{-6}$ & $1.6 \times 10^{-6}$ \\
upper fluid viscosity & $\nu_{U}$ & $\mathrm{~m}^{2} \mathrm{~s}^{-1}$ & $1.2 \times 10^{-6}$ & $1.2 \times 10^{-6}$ \\
lower fluid viscosity & $\nu_{L}$ & $\mathrm{~m}^{2} \mathrm{~s}^{-1}$ & $1 \times 10^{-6}$ & $1 \times 10^{-6}$ \\
upper fluid depth & $d_{U}$ & $\mathrm{~m}$ & 0.192 & 0.192 \\
lower fluid depth, initial & $d_{L}$ & $\mathrm{~m}^{-1}$ & 0.178 & 0.178 \\
rain interfacial tension & $\sigma_{r}$ & $\mathrm{~N} \mathrm{~m} \mathrm{~s}^{-1}$ & $2.2-2.4 \times 10^{-2}$ & $2.6 \times 10^{-2}$ \\
rain rate & $R$ & $\mathrm{~m} \mathrm{~s}^{-1}$ & $3.4-4.6 \times 10^{-4}$ & $4.5 \times 10^{-4}$ \\
rain buoyancy flux & $F$ & $\mathrm{~m}^{2} \mathrm{~s}^{-3}$ & $1.35-110 \times 10^{-6}$ & $-4.5 \times 10^{-5}$ \\
rain drop radius & $r_{r}$ & $\mathrm{~m}^{-1}$ & $\sim 10^{-3}$ & $\sim 10^{-3}$ \\
plume velocity & $w$ & $\mathrm{~m} \mathrm{~s}^{-1}$ & $3.4-14.3 \times 10^{-3}$ & $<4 \times 10^{-4}$ \\
\hline Dimensionless Parameter & Definition & Experiment & Experiment & Early Core \\
& & Type 1 & Type 2 & $($ Nominal) \\
\hline Density Ratio, $\epsilon$ & $\Delta \rho / \rho_{L}$ & $0.3-25 \times 10^{-3}$ & -0.01 & $\sim 4.6$ \\
Rayleigh Number, $R a_{F}$ & $F d_{L}^{4} / \nu_{L}^{3}$ & $1.35-113 \times 10^{9}$ & $-4.5 \times 10^{10}$ & $\sim 10^{29}$ \\
Plume Reynolds Number, $R e$ & $w d_{L} / \nu_{L}$ & $6-25 \times 10^{2}$ & $<2 \times 10^{1}$ & $\sim 2 \times 10^{7}$ \\
Magnetic Reynolds Number, $R m$ & $u d_{L} / \eta$ & na & na & $\sim 2 \times 10^{3}$ \\
\hline
\end{tabular}


Table 2: Table 2: Core Precipitation Properties (Nominal Values)

\begin{tabular}{|c|c|c|c|c|}
\hline Notation & Definition, Units & Early Earth & Mercury & Ganymede \\
\hline$r_{c}$ & convection radius, $\mathrm{km}$ & $3480^{a}$ & $500^{b}$ & $650^{j}$ \\
\hline$\Omega$ & rotation rate, $10^{-5} \mathrm{rad} \mathrm{s}^{-1}$ & $5^{c}$ & $0.124^{d}$ & $1.02^{l}$ \\
\hline$\eta$ & magnetic diffusivity, $\mathrm{m}^{2} \mathrm{~s}^{-1}$ & $0.8^{e}$ & $\sim 1$ & $0.7^{k}$ \\
\hline$\rho$ & average density, $10^{3} \mathrm{~kg} \mathrm{~m}^{-3}$ & $11^{a}$ & $7.7^{b}$ & $j$ \\
\hline$q_{s}$ & adiabatic heat flux, $\mathrm{mW} \mathrm{\textrm {m } ^ { - 2 }}$ & $100^{f}$ & $10^{b}$ & $4^{j}$ \\
\hline$q$ & CMB heat flux, $\mathrm{mW} \mathrm{m} \mathrm{m}^{-2}$ & $50-100^{f}$ & $3-10^{b}$ & $2-4^{l}$ \\
\hline$H_{c}$ & temperature scale height, km & $6500^{g}$ & $1400^{b}$ & $1100^{b}$ \\
\hline$g \Delta \rho / \rho$ & precipitation buoyancy, $\mathrm{m} \mathrm{s}^{-2}$ & $4.6^{h}$ & $0.4^{e}$ & $0.4^{e}$ \\
\hline$R$ & precipitation rate, $\mathrm{km} \mathrm{Gyr}^{-1}$ & $2.5^{n}$ & $50^{b}$ & $10^{j}$ \\
\hline$F$ & pricipitation buoyancy flux, $10^{-13} \mathrm{~m}^{2} \mathrm{~s}^{-3}$ & 6 & $4^{b}$ & $1.3^{m}$ \\
\hline$\nu$ & kinematic viscosity, $10^{-6} \mathrm{~m}^{2} \mathrm{~s}^{-1}$ & 1 & 1 & $1^{i}$ \\
\hline$k$ & thermal conductivity, $\mathrm{W} \mathrm{m} \mathrm{m}^{-1} \mathrm{k}^{-1}$ & $130^{f}$ & $120^{k}$ & $30^{j}$ \\
\hline
\end{tabular}

${ }^{a}$ PREM Dziewonski and Anderson (1981); ${ }^{b}$ Dumberry and Rivoldini (2015); ${ }^{c}$ Williams (2000); ${ }^{d}$ Ward et al. (1976); ${ }^{e}$ Hirose et al. (2013); ${ }^{f}$ Labrosse (2015); ${ }^{g}$ Vocadlo et al. (2003); ${ }^{h}$ PREM CMB buoyancy contrast; ${ }^{i}$ Perrialt et al. (2010); ${ }^{j}$ Christensen (2015); ${ }^{k}$ Deng et al. (2013); ${ }^{l}$ Hauk et al. (2006); ${ }^{m}$ Chen et al. (2008); ${ }^{n}$ O'Rourke and Stevenson, 2016. 
Figure 1: Precipitation-Driven Convection. Schematics of precipitation-driven convection in cooling planetary cores and laboratory analog. (a) Iron-snow; (b) Magnesium precipitation; (c) Laboratory apparatus, with the dashed box indicating the region imaged in Figure 2. M,P,C denote the mantle, the precipitation-dominated region, and the convectiondominated region, respectively. $r_{c}$ denotes convective region radius. Small arrows indicate precipitation directions; large arrows denote convection.

Figure 2: Rain-Driven Convection Experiments. In each case, raindrops (an aqueous solution) released at a constant and spatially uniform rate, fall through the upper liquid (silicone oil) into the lower liquid (another aqueous solution). In images (a) and (b) the blue-dyed rain is initially 1\% denser than the lower liquid (Type 1 experiments). This closeup sequence, corresponding to the dashed box in Figure 1c with time given in seconds after convective onset, shows the development of a two-phase emulsion layer, phase separation producing compositional plumes and turbulent convection, resulting in homogenization of the lower liquid. In images (c) and (d) the rain is initially 1\% less dense than the lower liquid (Type 2 experiments). In this case the rain products accumulate in the interfacial region and stratify the lower liquid. Experimental parameters are given in Table 1.

Figure 3: Development of a Rain-Driven Convection Region. This sequence shows the development of rain-driven convection in a Type 1 experiment with $0.1 \%$ excess rain density. (a): Onset of small scale compositional plumes; (b): Growth and turbulent merging of compositional plumes; (c): Large-scale convection in the lower layer; (d) mixing and homogenization. Time is given in seconds after rain injection begins.

Figure 4: Density Effects in Rain-Driven Convection. Comparison of rain-driven turbulent convection in Type 1 experiments with large and small density contrasts. (a): Convection with initial rain excess density of $0.03 \%$; (b): Convection with initial rain excess density of $2.5 \%$. Time is given in seconds after convective onset. 
Figure 5: Rayleigh Number-Reynolds Number Relation in Rain-Driven Convection. Reynolds number $R e$ based on plume descent speed versus flux Rayleigh number $R a_{F}$ from five Type 1 (dense rain) experiments, in which the Rayleigh number increases with increasing excess density of the rainfall. Symbol size is representative of 1 s.d. uncertainties in $R e$ and $R a_{F}$. Power law fit exponent and coefficient are given in the plot. Dashed line is a fit by Grossmann and Lohse (2002) to plume velocities in Rayleigh-Bénard experiments.

Figure 6: Dynamo Regimes from Magnesium Precipitation in Earth's Core. Dipole magnetic moment and local Rossby number for dynamo action in the totally fluid Earth's core (prior to inner core nucleation) driven by magnesium precipitation. Axes are core heat flux and precipitation rate. An adiabatic heat flux of $100 \mathrm{~mW} \mathrm{~m}^{-2}$ is assumed. Additional model parameters are given in Table 2.

Figure 7: Dynamo Regimes from Iron-snow Precipitation in Mercury. Magnetic Reynolds number $\mathrm{Rm}$ and local Rossby number $\mathrm{Ro}_{\ell}$ for dynamo action in the assumed totally fluid iron alloy core of Mercury, driven by iron-snow precipitation. Axes are ironsnow precipitation rate and convective radius. Total and adiabatic heat fluxes of 5 and 50 $\mathrm{mW} \mathrm{m}^{-2}$ are assumed. Additional model parameters are given in Table 2.

Figure 8: Dynamo Regimes from Iron-snow Precipitation in Ganymede. Dynamo regimes in the assumed totally fluid iron alloy core of Ganymede, driven by iron-snow precipitation. Contours are calculated local Rossby number $\mathrm{Ro}_{\ell}$. Axes are heat flux at the top of the convecting region and precipitation rate. An adiabatic heat flux of $4 \mathrm{~mW} \mathrm{~m}^{-2}$ is assumed. 
(c)

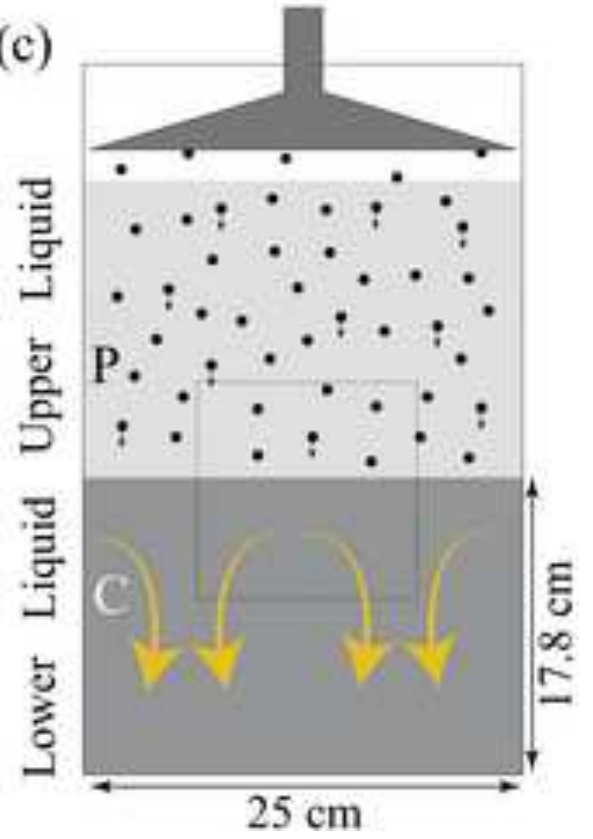

(a)

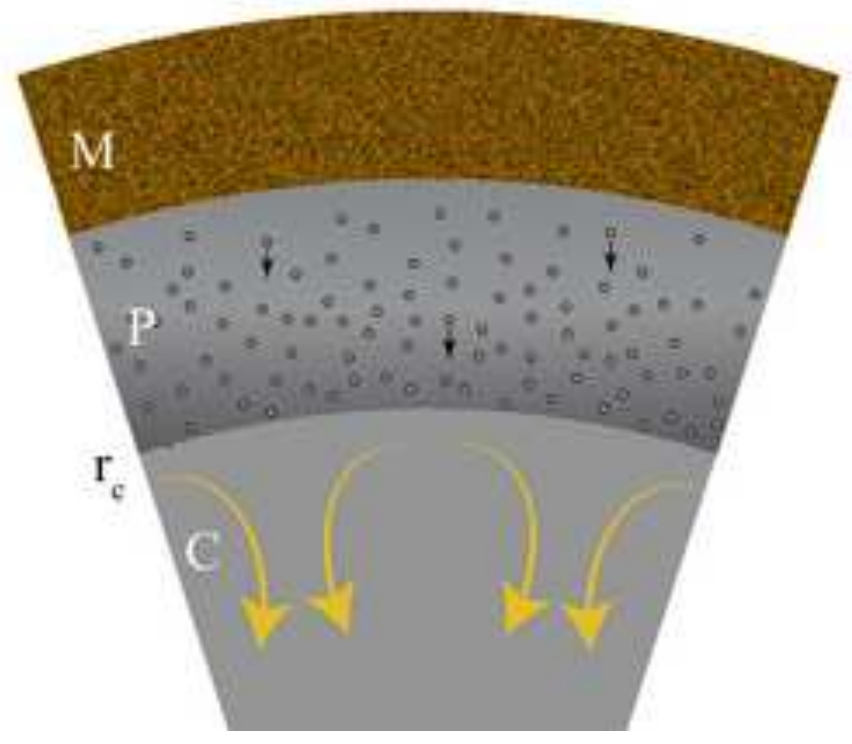

(b)

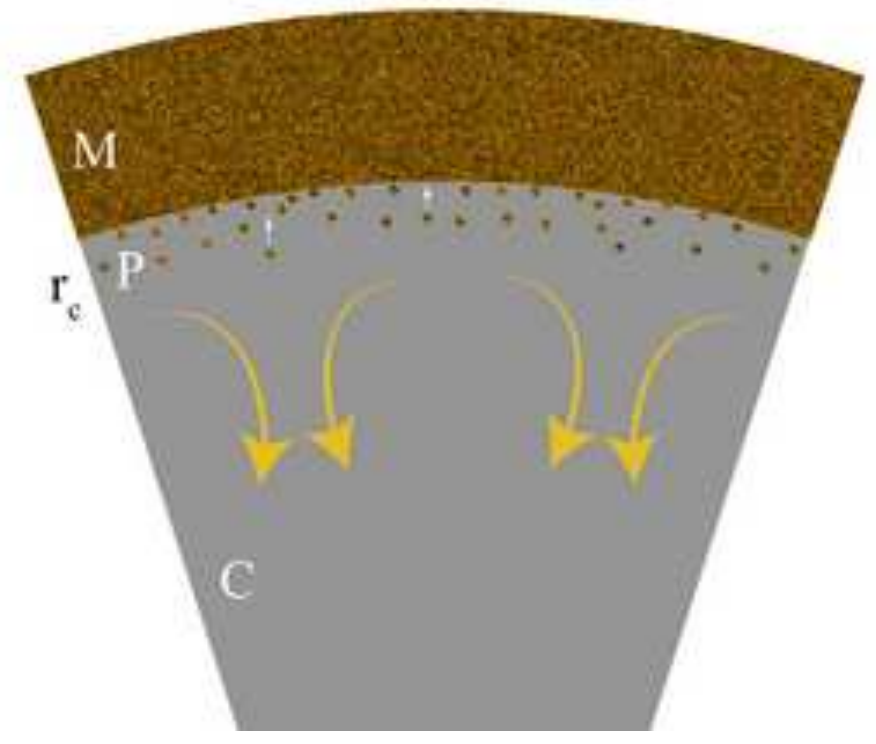

) 

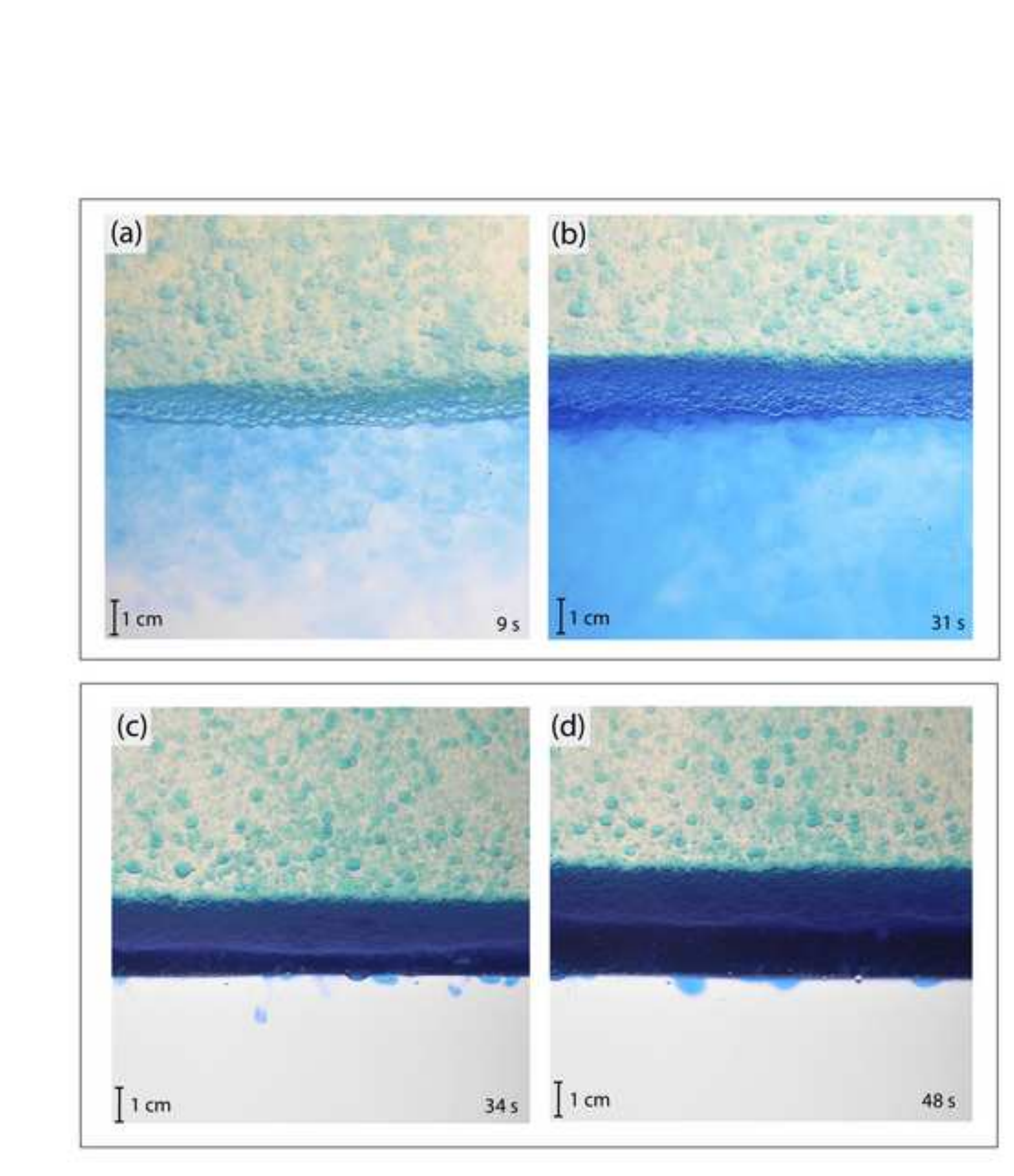

Figure 2

\section{Fing}

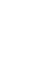
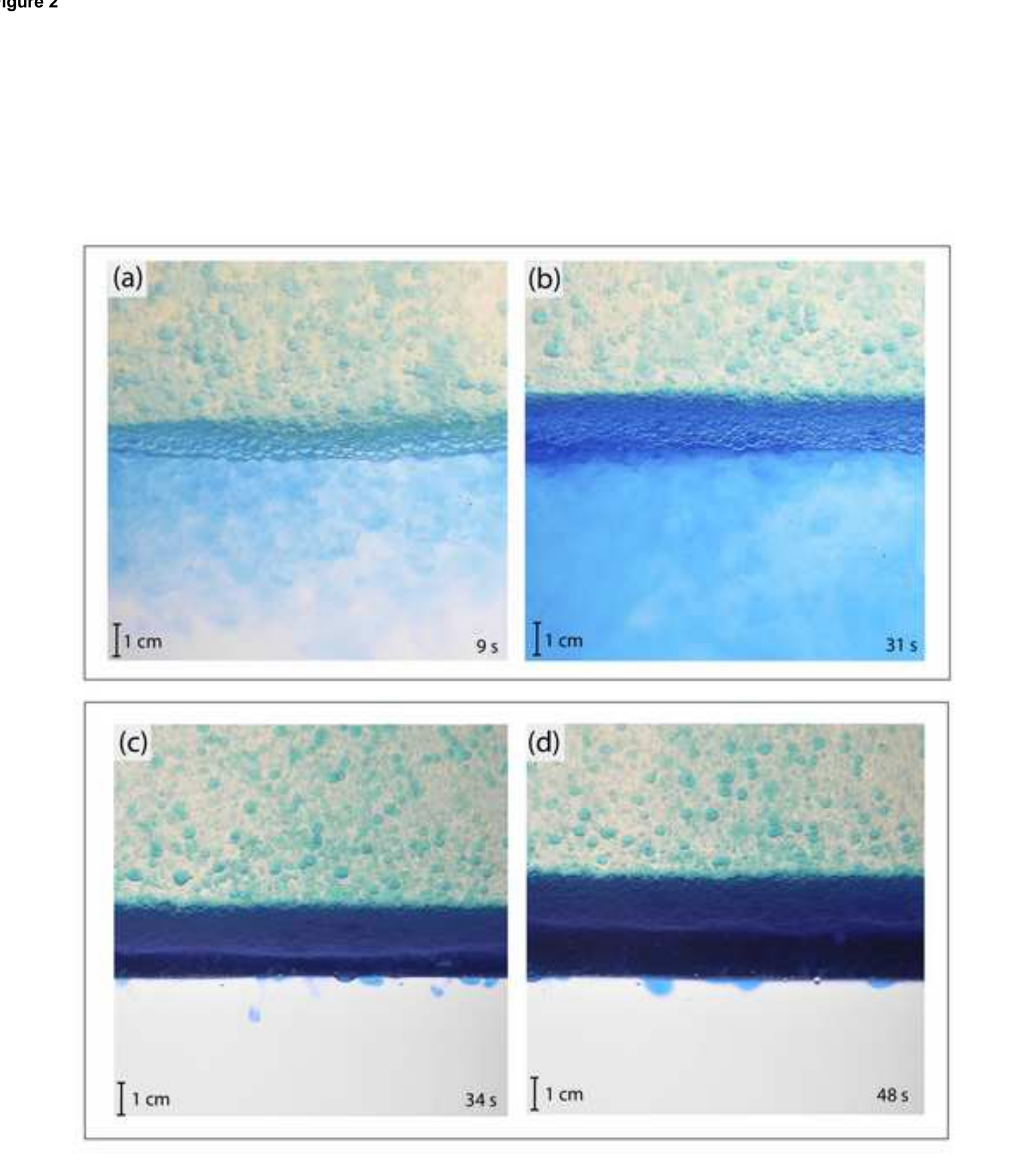


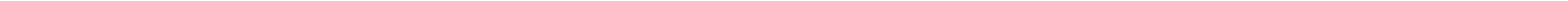




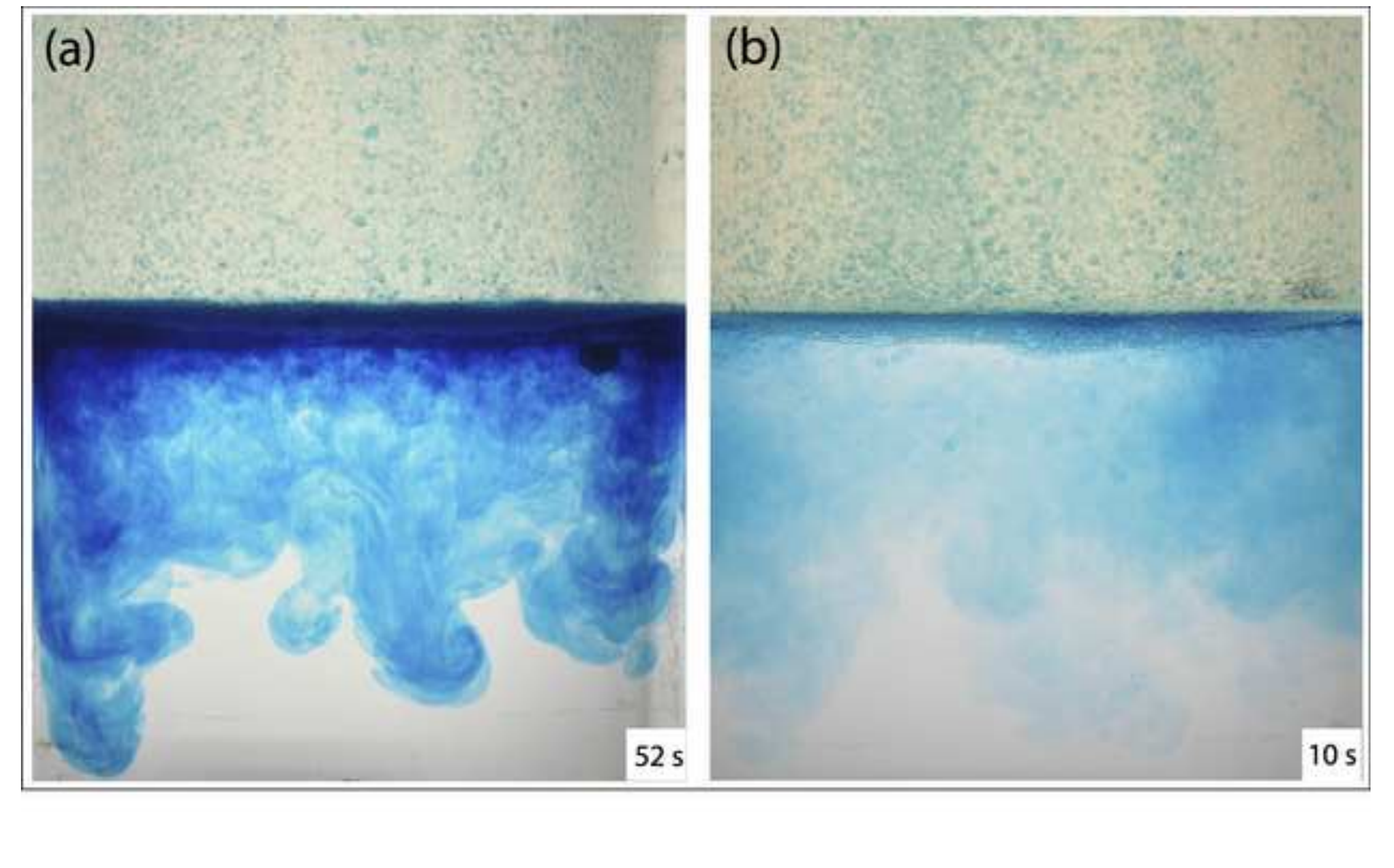

(b)

.

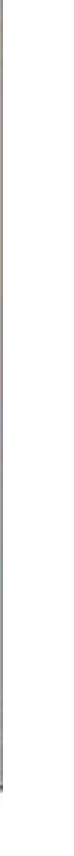

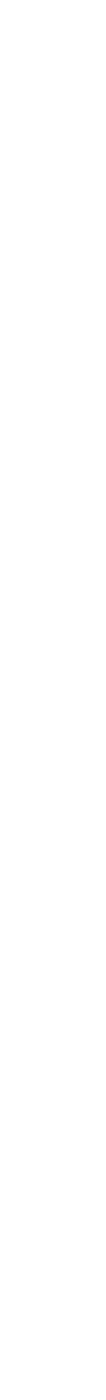

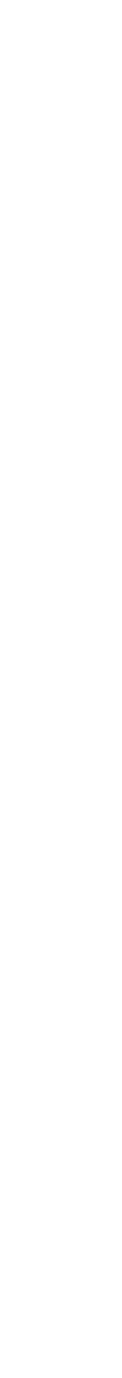

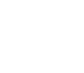




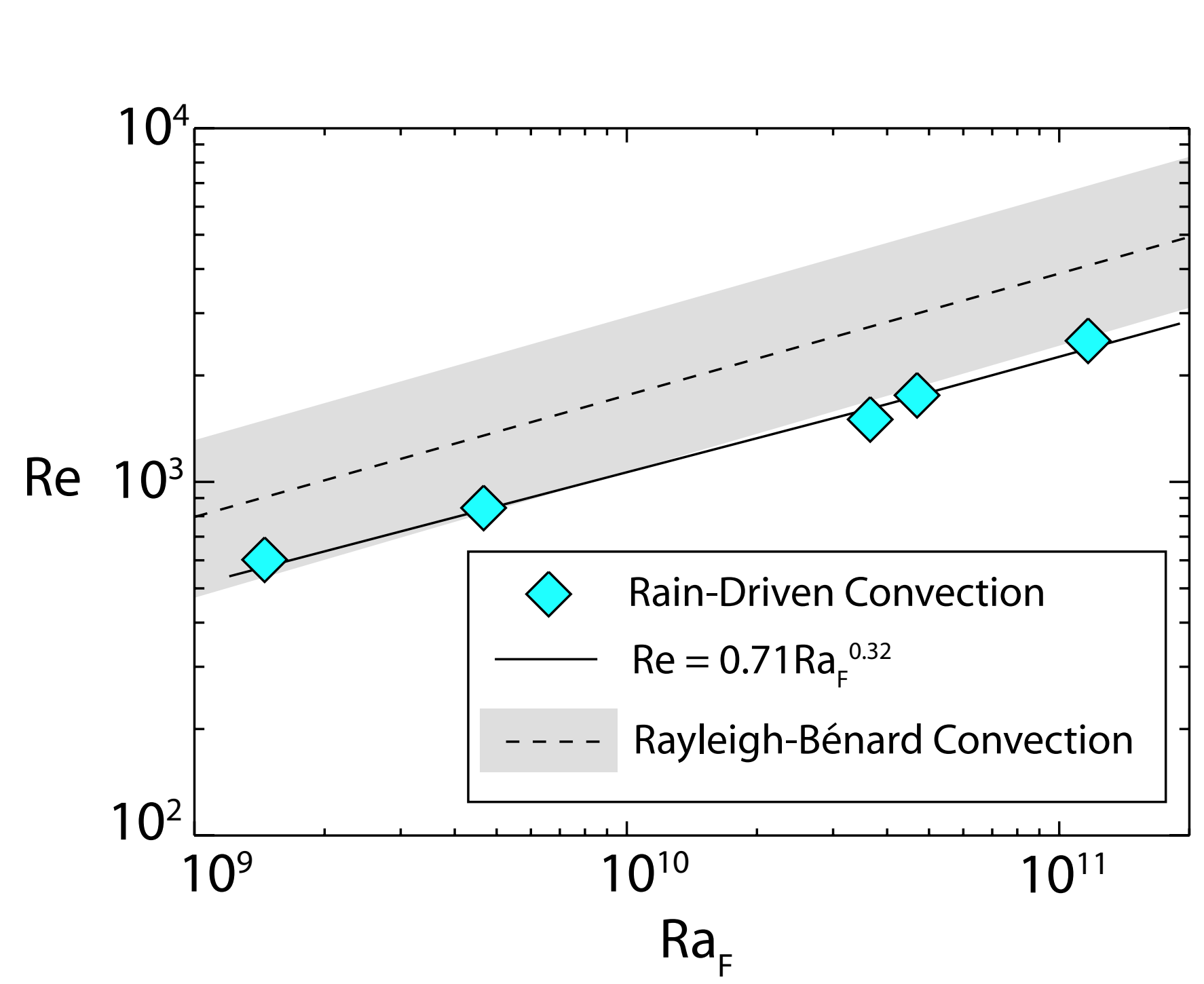

Figure 5

$$
\text { 栗 }
$$




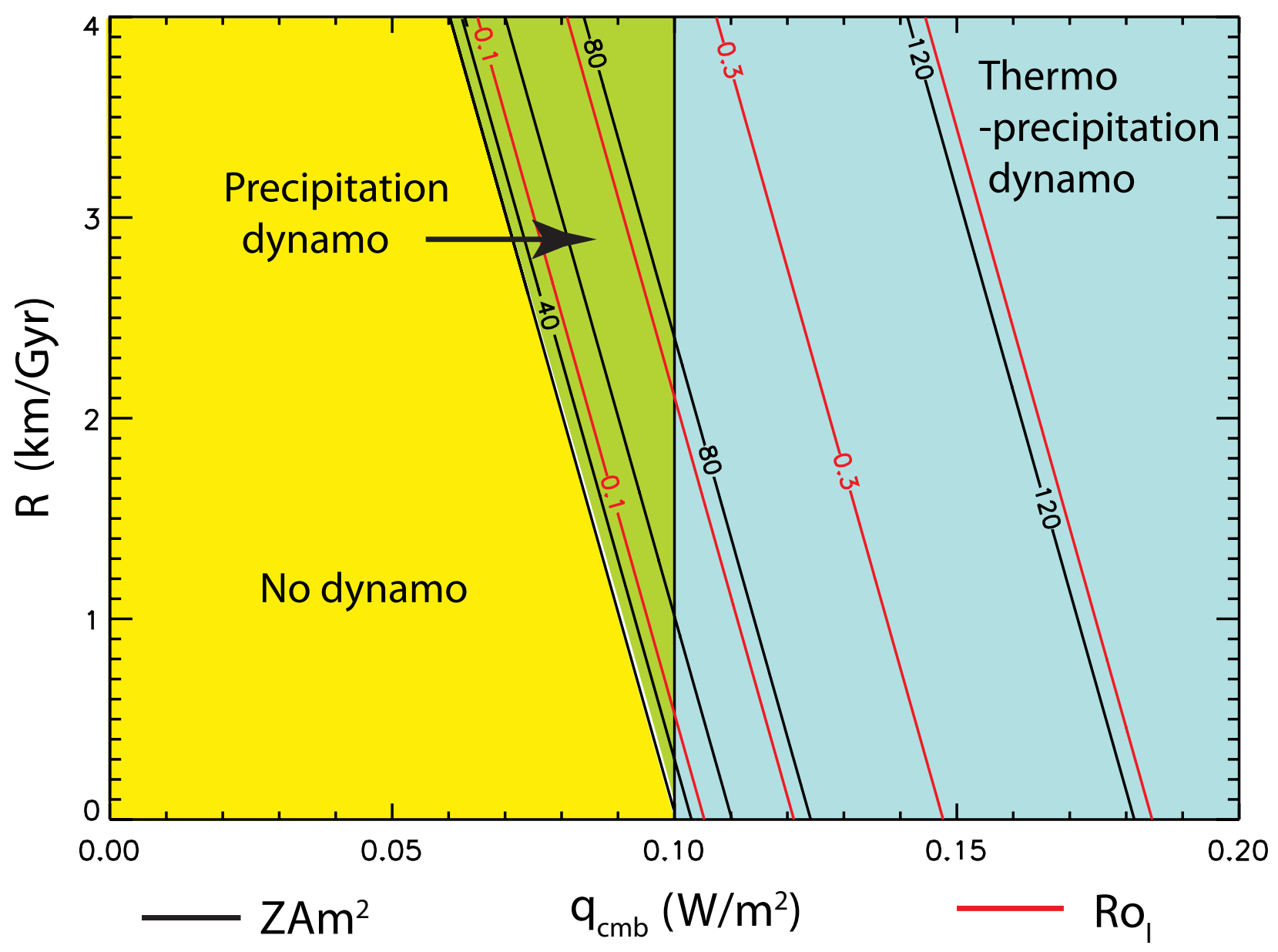




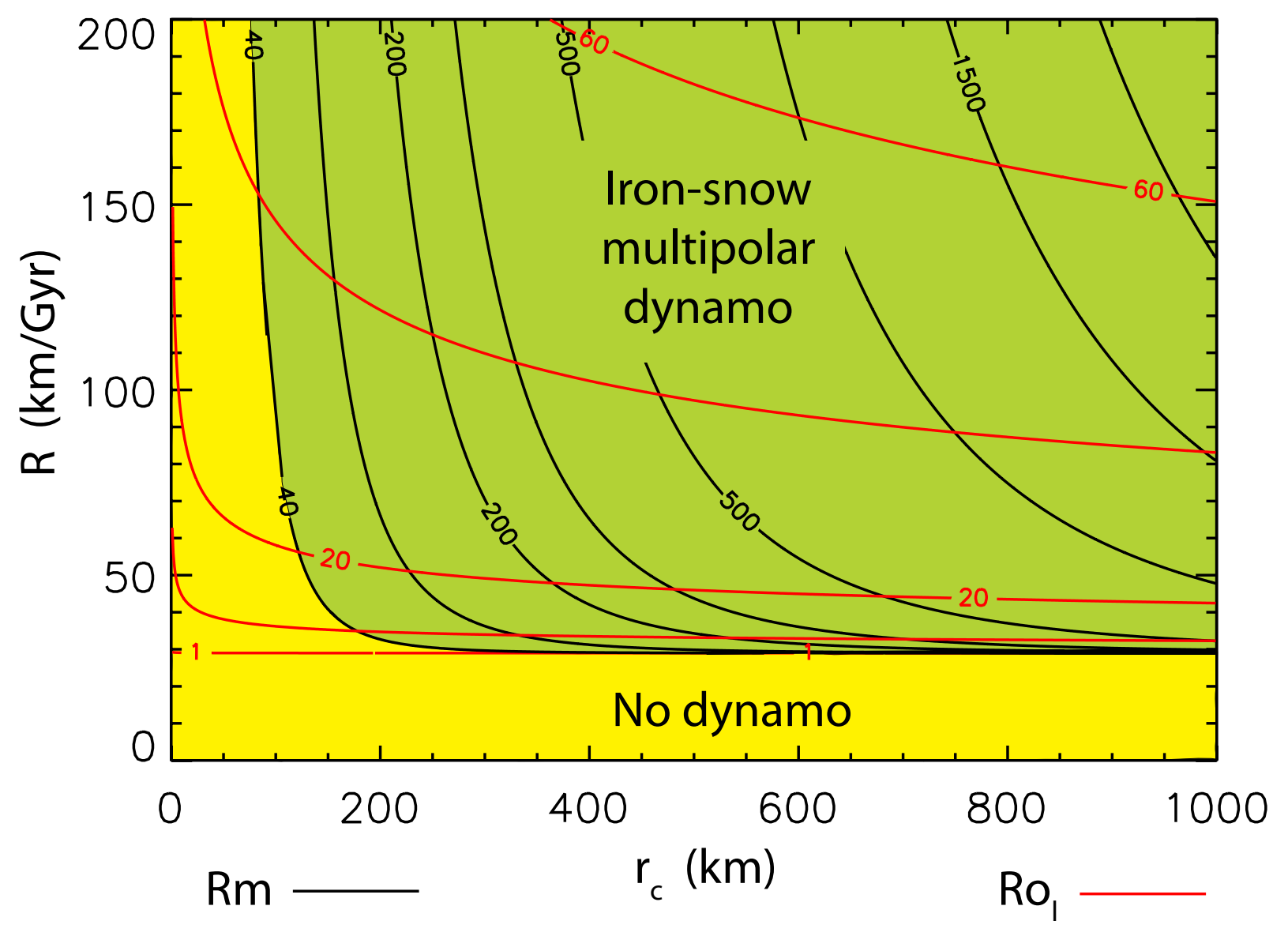




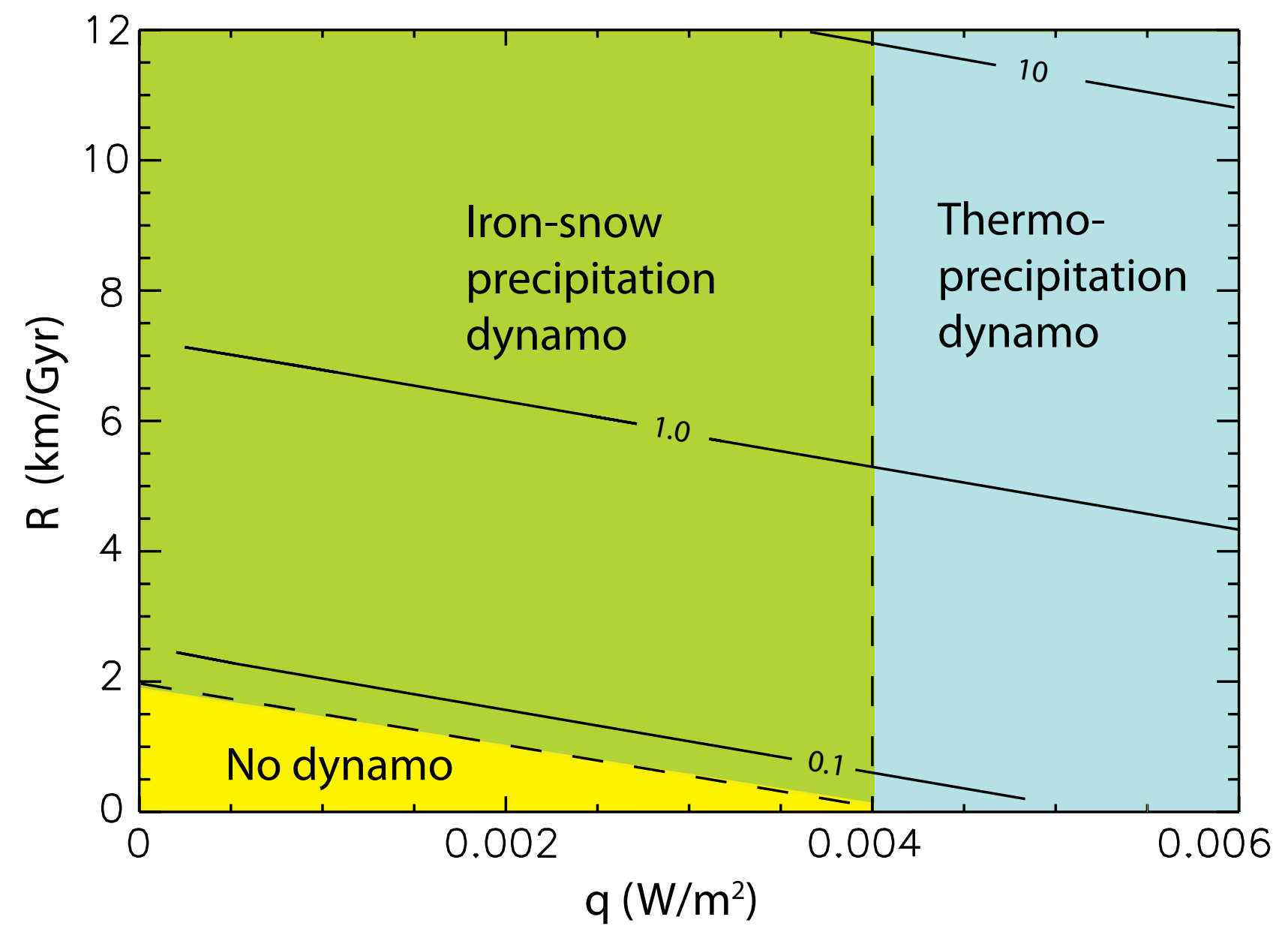

